\title{
Neural Network Estimator for Gas-Phase Ethylene Polymerization Process
}

\author{
Thanawat Kaewsanmuang ${ }^{1}$, Paisan Kittisupakorn ${ }^{1+}$ and David Banjerdpongchai ${ }^{2}$ \\ ${ }^{1}$ Department of Chemical Engineering, Chulalongkorn University, Bangkok, Thailand 10330 \\ ${ }^{2}$ Department of Electrical Engineering, Chulalongkorn University, Bangkok, Thailand 10330
}

\begin{abstract}
Ethylene concentration is a significant parameter in the polymerization process because it affects the molecular weight distribution of the polymer produced. However, there is a challenge to obtain a good estimation of ethylene concentration due to its highly nonlinear behavior and interaction among state variables. In this work, the neural network $(\mathrm{NN})$ has been applied to estimate the concentration of ethylene monomer with generic model control (GMC) controlling the reactor temperature. The neural network has been trained with backpropagation and Levenberg-Marquart techniques. Simulation results have shown that the neural network estimator can provide good estimates of the monomer concentration under nominal condition and disturbance cases.
\end{abstract}

Keywords: Polymerization process, Neural network, Estimator, Generic model control.

\section{Introduction}

The state feedback law is often based on the assumptions that all states are available for online measurement. Nonetheless, in practice, some of them may not be measurable due to lack of measurement or the high price of sensor devices. Therefore, measuring the states is not only difficulty but also the high cost of installation of these devices. Hence, estimators have been developed to reconstruct the state vector in order to estimate the states or variables. The ethylene polymerization process involves many challenging problems including nonlinear dynamic behavior, multivariable interactions between each state variable and unmeasurable state variables such as concentration, melt index and reaction rate. Estimation of unknown variables in polymerization processes is important for product quality control as well as in avoiding disruption in maintaining this quality [1-5].

For this work, we applied the neural network $(\mathrm{NN})$ to estimate the ethylene concentration in gas-phase ethylene polymerization reactor. $\mathrm{NN}$ is the most popular algorithms applied since its capability is promising and can be trained to learn the process based on historical data. Besides that, it provides high accuracy and consistent estimations even when changes occur in the process [6]. Then, generic model control (GMC) is used for control temperature in reactor.

Introduction is given in this section follow by the ethylene polymerization process model in section 2 . The neural network estimator and GMC design are discussed in section 3. Results are shown in section 4 while conclusion is in section 5 .

\section{Ethylene Polymerization Process Model}

Polyethylene is considered the world largest produced synthetic commodity polymer. The polyethylene reactor process is depicted in Fig. 1.

\footnotetext{
+ Corresponding author. Tel.: +662 2186878; fax: +662 2186877.
}

E-mail address: paisan.k@chula.ac.th. 


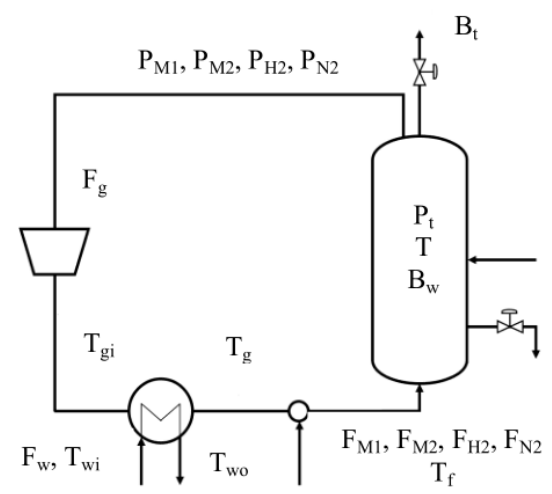

Fig. 1: Schematic of the polyethylene reactor.

The reactor is illustrated in Fig. 1 where the feed gas is combined with the recycled gas before entering the fluidized bed reactor. Another part of the fresh gas is used to introduce the Ziegler-Natta catalyst. Four major components are fed to the reactor. The gaseous species are Ethylene, Butene, hydrogen and nitrogen. In this process, the reactor temperature is controlled by manipulating the feed temperature of the cooling water. The process model was developed by McAuley et al (1990), and is given below.

$$
\begin{gathered}
V_{g} \frac{d C_{M 1}}{d t}=F_{M 1}-x_{M 1} B_{t}-R_{M 1} \\
V_{g} \frac{d C_{M 2}}{d t}=F_{M 2}-x_{M 2} B_{t}-R_{M 2} \\
V_{g} \frac{d C_{H}}{d t}=F_{H}-x_{H} B_{t} \\
V_{g} \frac{d C_{N}}{d t}=F_{N}-x_{N} B_{t} \\
\frac{d Y_{c}}{d t}=F_{c} a_{c}-k_{d} Y_{c}-O_{p} Y_{c} / B_{w} \\
\left(M_{r} C p_{r}+B_{w} C p_{p}\right) \frac{d T}{d t}=H F+H G-H R-H T-H P \\
M_{g} C p_{g} \frac{d T_{g}}{d t}=F_{g} C p_{g}\left(T_{g i}-T_{g}\right)+F_{w} C p_{w}\left(T_{w i}-T_{w o}\right) \\
P_{t}=\left(C_{M 1}+C_{M 2}+C_{H}+C_{N}\right) R T \\
T_{g}=\left(\frac{P_{t}}{P_{t}+\Delta P}\right)^{(1-\eta) / n} T \\
F_{w} C p_{w}\left(T_{w i}-T_{w o}\right)=0.5 U A\left[\left(T_{w o}+T_{w i}\right)-\left(T_{g i}+T_{g}\right)\right]
\end{gathered}
$$

where

$$
\begin{gathered}
H F=\left(F_{M 1} C p_{M 1}+F_{M 2} C p_{M 2}+F_{H} C p_{H}+F_{N} C p_{N}\right)\left(T_{f}-T_{r e f}\right) \\
H G=F_{g} C p_{g}\left(T_{g}-T_{r e f}\right) \\
H R=M_{w 1} R_{M 1} \Delta H_{r} \\
H T=\left(F_{g}+B_{t}\right) C p_{g}\left(T-T_{r e f}\right) \\
H P=O_{p} C p_{p}\left(T-T_{r e f}\right) \\
O_{p}=M_{w 1} R_{M 1}+M_{w 2} R_{M 2} \\
R_{M 1}=C_{M 1} Y_{c} k_{p 1} e^{-(E / R)\left(1 / T-1 / T_{r e f}\right)} \\
R_{M 2}=C_{M 2} Y_{c} k_{p 2} e^{-(E / R)\left(1 / T-1 / T_{r e f}\right)} \\
C p_{g}=\sum x_{i} C p_{i}
\end{gathered}
$$

The above equations are applied to develop the polymerization model in MATLAB to obtain the actual value that will be compared with the value from the proposed estimator.

\section{Estimator and Controller}


The reactor temperature is controlled by using the GMC control. The NN estimator is used estimate ethylene concentration. The scheme of system is show in Fig.2.

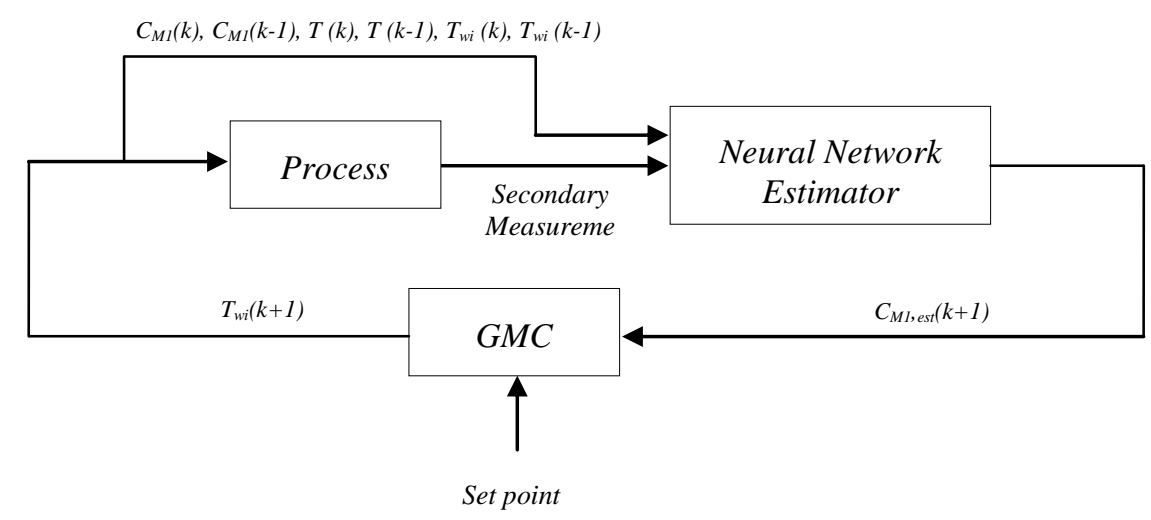

Fig. 2: Schematic of the NN estimator with GMC control.

\subsection{Neural Network (NN) Estimator}

Neural network $(\mathrm{NN})$ has been applied to nonlinear process modeling and control recently [7, 8]. They have the ability of learning the behavior of the process and the relationships between variables, without having a model of the phenomenological underlying laws.

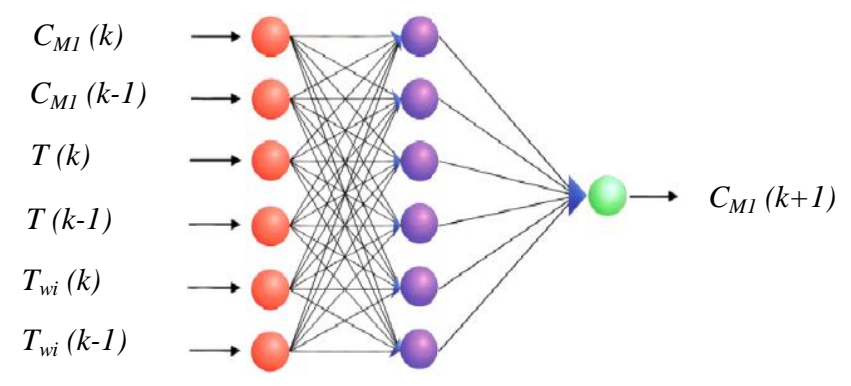

Fig. 3: Topology of the NN estimator.

In this work, feed forward neural network are used as estimators to estimate the ethylene concentration. Input of NN including ethylene concentration $\left(C_{M I}\right)$, temperature of reactor $(T)$ and inlet temperature of heat exchanger $\left(T_{w i}\right)$ are shown in Fig.3. The defined NN is trained with the Levenberg-Marquardt algorithm in the MATLAB neural network toolbox. After training, the trained neural networks are validated by validation data sets.

\subsection{Generic Model Control (GMC)}

This section presents the design of a controller to control the reactor temperature following a desired temperature trajectory. Let us consider a process based on the following model equations:

$$
\begin{gathered}
\frac{d x}{d t}=f(x, p, t)+g(x, t) u \\
y=h(x)
\end{gathered}
$$

where $x$ is a vector of state variables, $y$ is a vector of output variables, $u$ is a vector of input variables, $p$ is vector of process parameters, and $f, g$, and $h$ are non-linear function vectors. The general form of GMC algorithm can be written as

$$
\frac{d y}{d t}=K 1\left(y_{s p}-y\right)+K 2 \int_{0}^{t}\left(y_{s p}-y\right) d t
$$

The use of Eq. (22) forces $y$ toward its set point, $y_{\mathrm{sp}}$, with zero offset. If Eq. (21) is differentiated, and the Eq. (22) is substituted into the resulting equation, the GMC control law is 


$$
u=\frac{\left[K 1\left(y_{s p}-y\right)+K 2 \int_{0}^{t}\left(y_{s p}-y\right) d t-(d h / d x) f(x, d, t)\right]}{(d h / d x) g(x, t)}
$$

Applying, the GMC controller equation to the ethylene polymerization process is obtained as

$$
\left.T_{w i}=\left\{\frac{M_{w} C p_{w}\left\lfloor K 1\left(T_{s p}-T\right)+K 2 \int_{0}^{t}\left(T_{s p}-T\right) d t\right\rfloor-F_{g} C p_{g}\left(T_{g i}-T_{g}\right)}{F_{w} C p_{w}}\right\rfloor\left(F_{w} C p_{w}+0.5 U A\right)+0.5 U A\left(T_{g i}-T_{g}\right)\right\} / U A
$$

The GMC control response can be designed via the tuning parameters $K 1$ and $K 2$ based on the tuning curve given by Lee \& Sullivan (1988).

\section{Results}

$\mathrm{NN}$ estimator is applied to estimate the ethylene concentration in the ethylene polymerization process. The performances and robustness tests are carried out with two cases which are the nominal case and disturbance case. The controllers tuning parameters of GMC for reactor temperature used are given as $\mathrm{K} 1=$ 5 and $\mathrm{K} 2=0.04$.

\subsection{Nominal Case}

In this case, the $\mathrm{NN}$ estimators estimated ethylene concentration using the measured reactor temperature under the nominal condition. The estimated results are shown in Fig. 4.

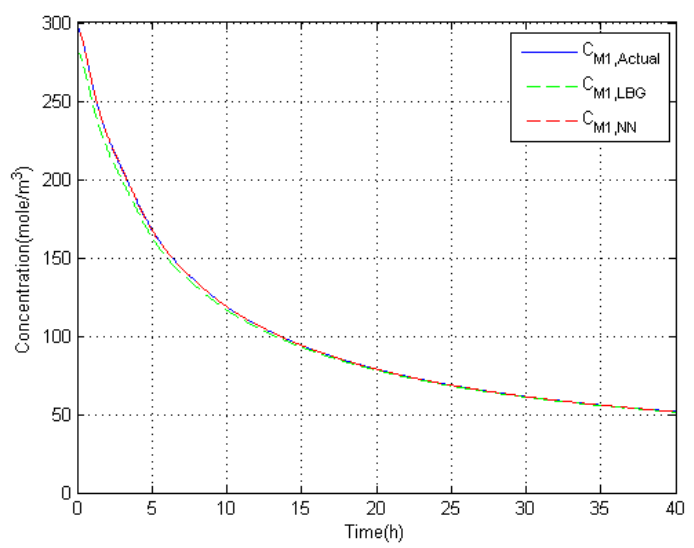

(a)

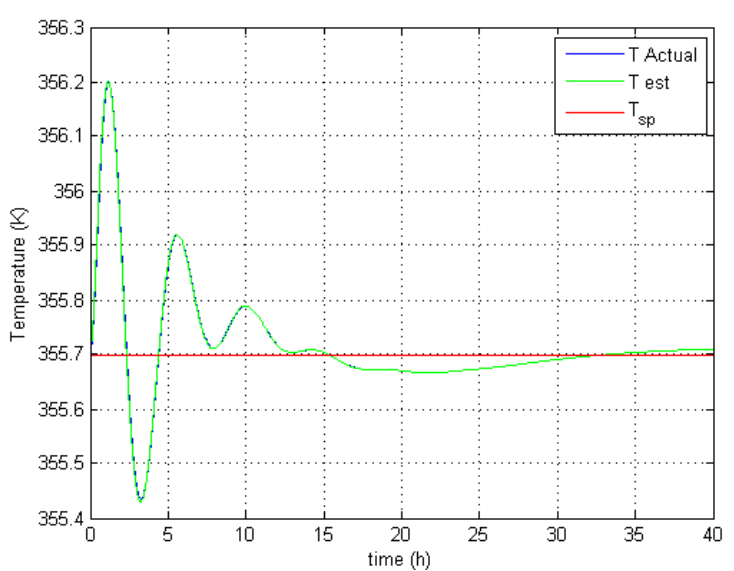

(b)

Fig. 4: The estimated ethylene concentration under nominal case: a) ethylene concentration estimated b) NN estimator with GMC

The NN estimator consists of 6 nodes of input layer, 4 nodes of one hidden layer and one node of output layer (6-4-1 configuration). From Fig.4 (a), the NN estimator gives good estimates of ethylene concentration when compared with the actual concentration and Luenberger observer from simulation of nonlinear model equations. Figure 4(b) shows reactor temperature which is controlled by GMC after apply NN estimator.

\subsection{Disturbance Case}

For the disturbance case, the flow rate of the ethylene $\left(F_{M I}\right)$ is changed by increasing its nominal operation values by $20 \%$. Figure 5 shows the results of the NN estimator for estimating the ethylene concentration with introduced $F_{M I}$ disturbance.

From Fig.5 (a), the NN estimator can give the estimates of the ethylene concentration accurately. Besides, GMC controller can still control reactor temperature converge to set point in Fig.5(b). It indicates that NN estimator can still provide good performances in the concentration estimation, even if, it receives effect from disturbance. 


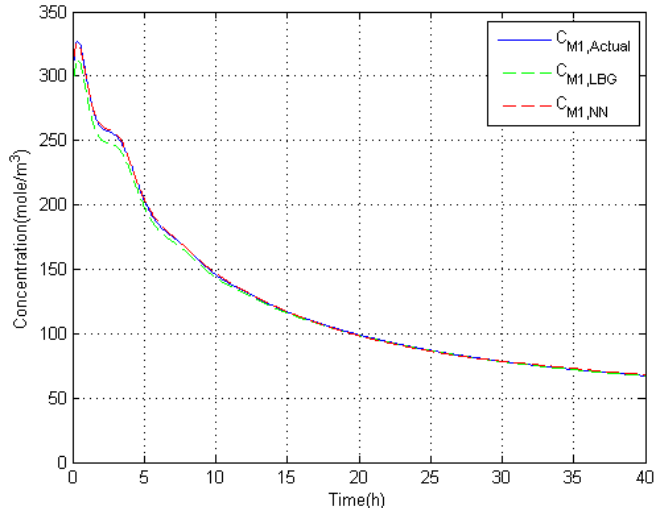

(a)

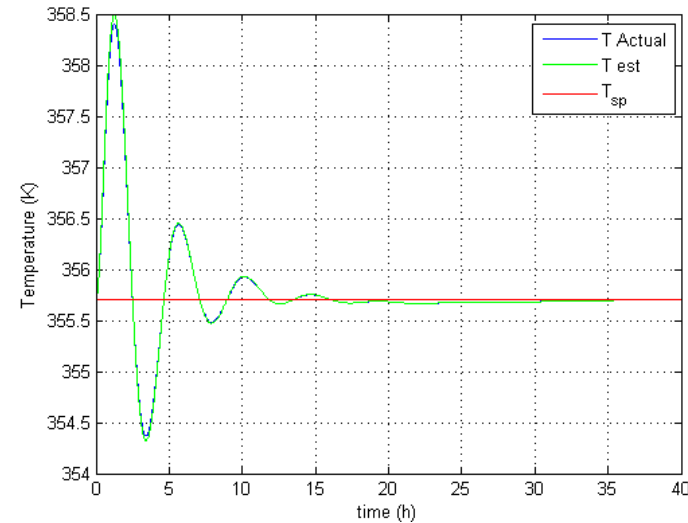

(b)

Fig. 5: The estimated ethylene concentration under the disturbance case: a) ethylene concentration estimated b) NN estimator with GMC

\section{Conclusions}

In this work, $\mathrm{NN}$ estimator is designed for estimating the ethylene concentration. Results of simulation have shown that $\mathrm{NN}$ estimator provides good estimates of ethylene concentration under nominal case and disturbance case with low error values when compared with the actual data. It should be noted that appropriate numbers of node, hidden layer, learning data and data range for training are required to achieve good models for this process. In future, the estimator will be applied to predict the other key features in a polymerization process such as, molecular weight distribution (MWD), heat transfer coefficient, melt index, and reaction rate by using the $\mathrm{NN}$ estimator.

\section{Acknowledgments}

The financial support to this work under The Institutional Research Grant (The Thailand Research Fund), IRG 5780014, and Chulalongkorn University, Contract No. RES_57_411_21_076.

\section{References}

[1] N. P. G. Salau, G. A. Neumann, J. O. Trierweiler and A. R. Secchi. Dynamic Behavior and Control in an Industrial Fluidized-Bed Polymerization Reactor. Ind. Eng. Chem. Res. 2008, 47: 6058-6069.

[2] A. Shamiri, M. A. Hussain, F. S. Mjalli, N. Mostoufi and S. Hajimolana. Dynamics and Predictive Control of Gas Phase Propylene Polymerization in Fluidized Bed Reactors. Chinese. J. Chem. Eng. 2013, 21(9): 1015-1029.

[3] G. A. Neumann, T. F. Finkler, N. S. M. Cardozo and A. R. Secchi. Parameter estimation for LLDPE gas-phase reactor models. Bra. J. Chem. Eng. 2007, 24(2): 267-275.

[4] R. Bindlish and J. B. Rawlings. Parameter Estimation for Industrial Polymerization Processes. AIChe. J. 2003, 49(8): 2071-2078

[5] A. Sirohi and K. Y. Choi. On-Line Parameter Estimation in a Continuous Polymerization Process. Ind. Eng. Chem. Res. 1996, 35: 1332-1343.

[6] R. Sharma, K. Singh, D. Singhal and R. Ghosh. Neural network applications for detecting process faults in packed towers. Chem. Eng. Process. 2004, 43: $841-847$.

[7] P. Kittisupakorn M. A. Hussain and J. Petcherdsak, Studies on the Use of Neural Networks in Nonlinear Control Strtegies. J. Chem. Eng. Jpn. 2001, 34(4): 453-465.

[8] W. Daosud, P. Thitiyasook, A. Arpornwichanop, P. Kittisupakorn and M. A. Hussain. Neural network inverse model-based controller for the control of a steel pickling process. Comput. Chem. Eng. 29(10): 2110-2119. 